\title{
Relationship between automated perimetry and Heidelberg retina tomograph, optic coherence tomography and laser polarimetry in moderate to severe glaucomatous eyes
}

\author{
Mehmet Emin Aslancı ${ }^{1}$, Mehmet Baykara ${ }^{2} \oplus$, Emre Güler $^{3} \oplus$, Özgür Bülent Timuçin ${ }^{4}$, Sami \\ Yılmaz $^{5} \odot$
}

${ }^{1}$ Department of Ophthalmology, Bursa City Hospital, Bursa, Turkey

${ }^{2}$ Department of Ophthalmology, Uludağ University School of Medicine, Bursa, Turkey

${ }^{3}$ Department of Ophthalmology, Türkiye Hospital, Istanbul, Turkey

${ }^{4}$ Department of Ophthalmology, Van Urartu Eye Center, Van, Turkey

${ }^{5}$ Department of Ophthalmology, Retina Eye Hospital, Bursa, Turkey

\section{ABSTRACT}

Objectives: To determine the correlations between the measurements obtained with Heidelberg retina tomograph III (HRT III), optic coherence tomography (OCT), and laser polarimetry (GDx) with the indices of automated perimetry (AP) in moderate and severe glaucoma patients.

Methods: Forty-nine eyes of 30 patients were included in the current study and were divided into two groups: 23 eyes with moderate and 26 eyes with severe glaucoma defined by Hodapp-Parrish-Anderson grading system. Pearson's correlation coefficients were used to evaluate the correlation between the indices of AP including mean deviation (MD) and pattern standard deviation (PSD), and structural parameters of the retinal nerve fiber layer (RNFL) and optic disc acquired by using three devices in both groups.

Results: In moderate glaucoma OCT and GDx measurements were not correlated to MD only the exception of inferior RNFL ( $\mathrm{r}=0.57, p=0.007$ and $\mathrm{r}=0.52, p=0.008$, respectively). Mild to moderate correlations were calculated between the structural parameters of HRT III and AP indices. In severe glaucoma, the most correlated measurements were obtained by OCT compared to the other devices. The correlations for MD were more powerful compared to PSD. Parameters based on the study of the RNFL showed stronger correlations than those of the optic nerve head. No devices showed significant correlations in patients with MD less than $-12 \mathrm{~dB}$.

Conclusions: OCT measurements showed the best correlations with the AP indices in both moderate and severe glaucoma patients. However, AP still seems to be more effective in the follow-up of glaucoma progression in more advanced glaucomatous damage.

Keywords: automated perimetry, Heidelberg retina tomograph, laser polarimetry, optic coherence tomography, glaucoma

laucoma is an optic neuropathy associated with the loss of retinal ganglion cells and their axons which create the retinal nerve fiber layer (RNFL) [1]. Several studies have shown that morphological 
changes of optic disc and defects in visual field (VF) are valuable indicators for diagnosis of glaucoma.

Although automated perimetry (AP) is the gold standard method for glaucoma diagnosis and progression documentation, morphological changes of optic disk and RNFL may precede VF defects in some cases [2]. Several studies have shown the efficacy of Heidelberg Retina Tomograph laser scan (HRT), laser polarimetry (GDx) and optic coherence tomography (OCT) for detecting glaucomatous changes of optic nerve and RNFL $[3,4]$.

Automated perimetry is useful to determine cases with moderate and advance glaucoma however it may be insufficient to detect patients in early stages $[5,6]$. In the other hand the recently imaging techniques have been reported to detect early stages of glaucoma [7]. In this study, we aimed to evaluate the structural parameters of the optic nerve head and RNFL obtained by using a HRT III, OCT and GDx with global indices of conventional AP and reveal the diagnostic precision of these devices for detecting and following subjects with moderate and severe primary open angle glaucoma (POAG).

\section{METHODS}

\section{Patients}

In this prospective study 49 eyes of 30 patients with POAG were included and were classified into two groups: 23 with moderate and 26 with severe glaucoma. Exclusion criteria were the presence of corneal opacity or cataract, refractive errors higher than -7.0 to +4.0 Diopters (D) sphere and/or 3.0 D cylinder, visual acuity less than $20 / 100$. The study was conducted in accordance with the ethical standards stated in the 1964 Declaration of Helsinki and approved by the Local Ethics Committee of the participating center. All patients were informed about the purpose of the study and provided their consent.

The definition of glaucoma was made by the findings of VF defects. The severity of the VF defect was graded as moderate, or severe visual field loss as defined by Hodapp-Parrish-Anderson (HPA) grading system criteria [8]. In this staging system, glaucomatous eyes were classified into three groups according to the mean deviation (MD) values (mild, MD $<-6$ $\mathrm{dB}$; moderate, $\mathrm{MD}>-6 \mathrm{~dB}$ and $<-12 \mathrm{~dB}$; severe, $\mathrm{MD}$
$>-12 \mathrm{~dB})$.

A complete ophthalmic examination and also measurements of AP, HRT III, OCT and GDx were obtained in all patients.

\section{Conventional Automatic Perimetry}

In all patients 30-2 central field test which uses the Swedish Interactive Threshold Algorithm (SITA) standard software program version 24-2 (Carl Zeiss Meditec, Oberkochen, Germany) on a Humphrey 750i automatic visual field analyzer (Carl Zeiss Meditec) was performed [9]. The test object was size III; the duration time was $200 \mathrm{~ms}$; and the background brightness was $31.5 \mathrm{asb}\left(10 \mathrm{~cd} / \mathrm{m}^{2}\right)$. Refractive errors and presbyopia were corrected before the examination. The test was accepted to be confidential when fixation loss, false-negative and false-positive rates were less than $30 \%$. Abnormality was evaluated using the instrument's software through calculating the global Indices of MD or pattern standard deviation (PSD).

\section{Confocal Scanning Laser Ophthalmoscope Imag- ing}

Confocal scanning laser ophthalmoscopy measurements were obtained with the HRT III (Heidelberg Engineering) as described previously [10]. The same examiner performed 3 sequential measurements and these were aligned to combine unique image. The margins of the optic disc were marked by the same examiner manually. Once the margins were marked, disc parameters were estimated automatically by the device. The evaluated parameters in our study were rim area, rim volume, linear cup to-disk ratio, cup-to-disk area ratio, rim-to-disk area ratio, RNFL cross-sectional area, mean RNFL thickness, cup area, cup volume, and mean cup depth.

\section{Optical Coherence Tomography}

One examiner performed the peripapillary RNFL thickness measurements by the Stratus TD OCT (Carl Zeiss Meditec) which were described previously [11]. The accepted images had at least 7 signal quality. Study protocols were carried out for RNFL thicknesses (mean, superior and inferior), central foveal thickness and total foveal volume.

\section{Laser Polarimetry}

Scanning Laser polarimetry measurements were 
obtained by using GDx VCC (Carl Zeiss Meditec Inc.). Details of the GDx-VCC operation have been described [12]. The device calculated the RNFL thicknesses for temporal, superior, nasal and inferior quadrants and nerve fiber indicator (NFI). Measurements with quality score grading less than 7 and scan score less than 70 were excluded.

\section{Statistical Analysis}

Statistical analyses were calculated using SPSS software (version 21.0, SPSS, Inc. Chicago, IL, USA). Kolmogorov-Smirnov test revealed that the data was normally distributed $(\mathrm{p}>0.05)$. The results are presented as the mean \pm the standard deviation (SD). The measurements between each group were compared by Paired $t$ test. Correlation of measured parameters obtained by OCT, HRT III and GDx VCC with MD and PSD was analyzed with Pearson correlation coefficient. $P$ value $<0.05$ was considered statistically significant.

\section{RESULTS}

A total of 49 eyes of 30 patients $(20$ males, 10 females) with POAG were evaluated. The mean age of the patients was $56.2 \pm 10.0$ years. As defined by HPA grading system 14 patients ( 23 eyes) had moderate

Table 1. Comparison of the measured parameters obtained by OCT, HRT III and GDx between the groups

$\begin{array}{ccc}\begin{array}{c}\text { Moderate POAG } \\ (\mathrm{n}=23)\end{array} & \begin{array}{c}\text { Severe POAG } \\ (\mathrm{n}=\mathbf{2 6})\end{array} & p \text { value } \\ \end{array}$

\begin{tabular}{|cccc}
\hline OCT parameters & & & \\
\hline RNFL superior $(\mu \mathrm{m})$ & $86.76 \pm 17.74$ & $57.80 \pm 15.45$ & $\mathbf{0 . 0 0 1}$ \\
\hline RNFL inferior $(\mu \mathrm{m})$ & $75.42 \pm 16.32$ & $54.76 \pm 14.12$ & $\mathbf{0 . 0 0 1}$ \\
\hline RNFL average $(\mu \mathrm{m})$ & $65.81 \pm 7.88$ & $51.72 \pm 9.08$ & $\mathbf{0 . 0 0 1}$ \\
\hline CFT $(\mu \mathrm{m})$ & $209 \pm 24$ & $175 \pm 16$ & $\mathbf{0 . 0 0 1}$ \\
\hline Total macular volume $(\mu \mathrm{m})$ & $5.83 \pm 0.43$ & $6.38 \pm 0.37$ & $\mathbf{0 . 0 0 1}$ \\
\hline GDx parameters & & & \\
\hline TSNIT average $(\mu \mathrm{m})$ & $45.08 \pm 2.50$ & $38.40 \pm 5.44$ & $\mathbf{0 . 0 0 1}$ \\
\hline RNFL superior $(\mu \mathrm{m})$ & $52.38 \pm 4.80$ & $39.35 \pm 8.26$ & $\mathbf{0 . 0 0 1}$ \\
\hline RNFL inferior $(\mu \mathrm{m})$ & $51.21 \pm 7.16$ & $41.76 \pm 9.17$ & $\mathbf{0 . 0 0 1}$ \\
\hline NFI & $47.04 \pm 10.45$ & $74.48 \pm 15.94$ & $\mathbf{0 . 0 0 1}$ \\
\hline HRT III parameters & & & \\
\hline Cup area $\left(\right.$ mm $\left.^{2}\right)$ & $1.03 \pm 0.40$ & $1.34 \pm 0.42$ & $\mathbf{0 . 0 1 7}$ \\
\hline Rim area $\left(\right.$ mm $\left.^{2}\right)$ & $0.96 \pm 0.30$ & $0.76 \pm 0.21$ & $\mathbf{0 . 0 2 6}$ \\
\hline Rim volume $\left(m m^{3}\right)$ & $0.17 \pm 0.07$ & $0.14 \pm 0.07$ & 0.057 \\
\hline Cup/Discarea & $0.53 \pm 0.17$ & $0.63 \pm 0.13$ & $\mathbf{0 . 0 2 7}$ \\
\hline Cup/Discarea $($ linear $)$ & $0.70 \pm 0.12$ & $0.79 \pm 0.09$ & $\mathbf{0 . 0 1 0}$ \\
\hline Average cup depth $(\mathrm{mm})$ & $0.27 \pm 0.08$ & $0.37 \pm 0.15$ & $\mathbf{0 . 0 0 9}$ \\
\hline Maximum cupdepth $(\mathrm{mm})$ & $0.57 \pm 0.14$ & $0.76 \pm 0.26$ & $\mathbf{0 . 0 0 3}$ \\
\hline Cup shape measure & $-0.060 \pm 0.002$ & $-0.020 \pm 0.001$ & $\mathbf{0 . 0 4 1}$ \\
\hline RNFL average $(\mu \mathrm{m})$ & $0.14 \pm 0.05$ & $0.10 \pm 0.07$ & $\mathbf{0 . 0 2 7}$ \\
\hline
\end{tabular}

Values are given as mean \pm standard deviation. POAG $=$ Primary open angle glaucoma, OCT $=$ Optical coherence tomography, HRT $=$ Heidelberg retina tomograph, GDx $=$ Laser polarimetry, RNFL = Retinal nerve fiber layer, CFT $=$ Central foveal thickness, TSNIT $=$ Temporal-superior-nasal-inferior-temporal, NFI $=$ Nerve fiber indicator. $p<0.05$ indicates statistically significance difference. 
POAG, 16 patients (26 eyes) had severe POAG. There were no significant differences in age and sex among the two groups ( $p=0.31$ and $p=0.28$, respectively). Comparison of the measured parameters obtained by OCT, HRT III and GDx between the groups are shown in Table 1. All the measured parameters among the two groups were significantly higher for OCT and GDx $(p<0.001)$ and statistically significant for HRT III $(p<0.05)$.

Any correlations between the measured parameters and the AP indices of the moderate glaucoma patients are shown in Table 2. OCT and GDx measurements were not correlated to MD only the exception of inferior RNFL for both devices $(\mathrm{r}=0.57, p$
$=0.007$ and $\mathrm{r}=0.52, p=0.008$, respectively). In addition there was no significant correlation between these devices and PSD. Regarding the measurements of HRT III, significant correlations were found between cup area, cup/disc area, cup/disc area (linear) and maximum cup depth and MD $(\mathrm{r}=-0.50, p=0.02$, $\mathrm{r}=-0.41, p=0.04 ; \mathrm{r}=-0.44, p=0.03 ; \mathrm{r}=-0.43, p=$ 0.05 , respectively). Additionally, cup area, cup/disc area, cup/disc area (linear) and average RNFL were significantly correlated to PSD $(\mathrm{r}=-0.59, p=0.005, \mathrm{r}$ $=-0.54, p=0.01, \mathrm{r}=-0.50, p=0.02 ; \mathrm{r}=0.45, p=0.04$, respectively).

Correlations between the measured parameters and the AP indices of the severe glaucoma patients are

Table 2. Correlations between the measured parameters and the MD-PSD of the moderate POAG

\begin{tabular}{|c|c|c|c|c|}
\hline & \multicolumn{2}{|c|}{$\operatorname{MD}(n=23)$} & \multicolumn{2}{|c|}{$\operatorname{PSD}(n=23)$} \\
\hline & $\mathbf{r}$ & $p$ value & $\mathbf{r}$ & $p$ value \\
\hline \multicolumn{5}{|l|}{ OCT parameters } \\
\hline RNFL average $(\mu \mathrm{m})$ & 0.20 & 0.38 & -0.07 & 0.75 \\
\hline RNFL superior $(\mu \mathrm{m})$ & 0.10 & 0.67 & -0.05 & 0.81 \\
\hline RNFL inferior $(\mu \mathrm{m})$ & 0.57 & 0.007 & -0.05 & 0.83 \\
\hline $\mathrm{CFT}(\mu \mathrm{m})$ & 0.43 & 0.051 & -0.19 & 0.41 \\
\hline Total macular volume $(\mu \mathrm{m})$ & 0.20 & 0.41 & 0.05 & 0.84 \\
\hline \multicolumn{5}{|l|}{ GDx parameters } \\
\hline TSNIT average $(\mu \mathrm{m})$ & 0.22 & 0.36 & 0.21 & 0.35 \\
\hline RNFL superior $(\mu \mathrm{m})$ & 0.18 & 0.45 & -0.16 & 0.49 \\
\hline RNFL inferior $(\mu \mathrm{m})$ & 0.52 & 0.008 & 0.20 & 0.39 \\
\hline NFI & -0.14 & 0.54 & 0.06 & 0.78 \\
\hline \multicolumn{5}{|l|}{ HRT III parameters } \\
\hline Cup area $\left(\mathrm{mm}^{2}\right)$ & -0.50 & 0.02 & -0.59 & 0.005 \\
\hline $\operatorname{Rim}$ area $\left(\mathrm{mm}^{2}\right)$ & 0.27 & 0.23 & 0.04 & 0.86 \\
\hline Rim volume $\left(\mathrm{mm}^{3}\right)$ & 0.27 & 0.23 & 0.15 & 0.50 \\
\hline Cup/Disc area & -0.41 & 0.04 & -0.54 & 0.01 \\
\hline Cup/Disc area (linear) & -0.44 & 0.03 & -0.50 & 0.02 \\
\hline Average cup depth (mm) & -0.34 & 0.12 & -0.30 & 0.18 \\
\hline Maximum cup depth (mm) & -0.43 & 0.05 & -0.16 & 0.49 \\
\hline Cup shape measure & -0.03 & 0.88 & -0.35 & 0.12 \\
\hline RNFL average $(\mu \mathrm{m})$ & -0.06 & 0.79 & 0.45 & 0.04 \\
\hline
\end{tabular}

$\mathrm{POAG}=$ Primary open angle glaucoma, $\mathrm{OCT}=$ Optical coherence tomography, $\mathrm{HRT}=$ Heidelberg retina tomograph, $\mathrm{GDx}=$ Laser polarimetry, RNFL $=$ Retinal nerve fiber layer, $\mathrm{CFT}=$ Central foveal thickness, TSNIT $=$ Temporalsuperior-nasal-inferior-temporal, NFI $=$ Nerve fiber indicator, MD $=$ median deviation, $\mathrm{PSD}=$ pattern standard deviation. $p<0.05$ indicates statistically significance difference. 
shown in Table 3. The RNFL (average, superior and inferior) as measured by OCT was significantly correlated with $\mathrm{MD}(\mathrm{r}=0.74, p=0.001 ; \mathrm{r}=0.68, p=$ $0.001 ; \mathrm{r}=0.70, p=0.001$, respectively). However, no significant correlation was found between OCT measurements and PSD $(p>0.05)$. The inferior RNFL and NFI obtained by GDx were significantly correlated with MD $(\mathrm{r}=0.66, p=0.001 ; \mathrm{r}=-0.66, p=0.001$, respectively). Similarly no significant correlation was found between GDx measurements and PSD ( $p>$ 0.05). Regarding the measurements of HRT III, significant correlations were found between cup area, cup/disc area, cup/disc area (linear), average and maximum cup depth and cup shape measure and MD ( $\mathrm{r}=$
$-0.51, p=0.01 ; \mathrm{r}=-0.45, p=0.03 ; \mathrm{r}=-0.44, p=0.03 ;$ $\mathrm{r}=-0.57, p=0.003 ; \mathrm{r}=-0.61, p=0.001$, respectively). Additionally, cup area and rim volume were significantly correlated to PSD ( $\mathrm{r}=-0.54, p=0.005 ; \mathrm{r}=$ $0.47, p=0.02$, respectively).

In patients with MD less than $-20 \mathrm{~dB}$ (12 patients), the measurements of OCT, GDx and HRT III did not show any significant correlation with MD and $\operatorname{PSD}(p$ $>0.05$ ) (Table 4).

\section{DISCUSSION}

In this study we compared the structural parame-

Table 3. Correlations between the measured parameters and the MD-PSD of the severe POAG

\begin{tabular}{|ccccc}
\hline & \multicolumn{2}{c}{ MD $(\mathbf{n}=\mathbf{2 6})$} & \multicolumn{2}{c}{ PSD (n= 26) } \\
& $\mathbf{r}$ & $\boldsymbol{p}$ value & $\mathbf{r}$ & $\boldsymbol{p}$ value \\
\hline OCT parameters & & & & \\
\hline RNFL average $(\mu \mathrm{m})$ & 0.74 & $\mathbf{0 . 0 0 1}$ & 0.16 & 0.45 \\
\hline RNFL superior $(\mu \mathrm{m})$ & 0.68 & $\mathbf{0 . 0 0 1}$ & 0.18 & 0.40 \\
\hline RNFL inferior $(\mu \mathrm{m})$ & 0.70 & $\mathbf{0 . 0 0 1}$ & 0.19 & 0.36 \\
\hline CFT $(\mu \mathrm{m})$ & 0.35 & 0.08 & 0.08 & 0.71 \\
\hline Total macular volume $(\mu \mathrm{m})$ & 0.33 & 0.11 & 0.14 & 0.50 \\
\hline GDx parameters & & & & \\
\hline TSNIT average $(\mu \mathrm{m})$ & 0.43 & $\mathbf{0 . 0 4 5}$ & 0.04 & 0.85 \\
\hline RNFL superior $(\mu \mathrm{m})$ & 0.47 & $\mathbf{0 . 0 3 2}$ & -0.13 & 0.53 \\
\hline RNFL inferior $(\mu \mathrm{m})$ & 0.66 & $\mathbf{0 . 0 0 1}$ & 0.40 & 0.05 \\
\hline NFI & -0.66 & $\mathbf{0 . 0 0 1}$ & -0.07 & 0.74 \\
\hline HRT III parameters & & & & \\
\hline Cup area $\left(\mathrm{mm}^{2}\right)$ & -0.51 & $\mathbf{0 . 0 1}$ & -0.54 & 0.005 \\
\hline Rim area $\left(\mathrm{mm}^{2}\right)$ & 0.34 & 0.09 & 0.04 & 0.79 \\
\hline Rim volume $\left(\mathrm{mm}^{3}\right)$ & 0.20 & 0.34 & 0.47 & 0.02 \\
\hline Cup/Disc area & -0.45 & 0.03 & -0.28 & 0.35 \\
\hline Cup/Disc area $($ linear) & -0.44 & 0.03 & -0.35 & 0.06 \\
\hline Average cup depth $(\mathrm{mm})$ & -0.57 & 0.003 & -0.26 & 0.06 \\
\hline Maximum cup depth $(\mathrm{mm})$ & -0.41 & 0.035 & -0.34 & 0.06 \\
\hline Cup shape measure & -0.61 & 0.001 & -0.38 & 0.06 \\
\hline RNFL average $(\mu \mathrm{m})$ & -0.01 & 0.96 & 0.156 & 0.46 \\
\hline POAG & Prinary op & & & \\
\hline
\end{tabular}

POAG $=$ Primary open angle glaucoma, OCT $=$ Optical coherence tomography, HRT $=$ Heidelberg retina tomograph, GDx $=$ Laser polarimetry, RNFL $=$ Retinal nerve fiber layer, CFT $=$ Central foveal thickness, TSNIT $=$ Temporalsuperior-nasal-inferior-temporal, NFI = Nerve fiber indicator, MD =median deviation, PSD = pattern standard deviation. $p<0.05$ indicates statistically significance difference. 
Table 4. Correlations between the measured parameters and the MD-PSD of the patients with MD less than $-20 \mathrm{~dB}$

\begin{tabular}{|c|c|c|c|c|}
\hline & \multicolumn{2}{|c|}{$\operatorname{MD}(n=12)$} & \multicolumn{2}{|c|}{$\operatorname{PSD}(n=12)$} \\
\hline & $\mathbf{r}$ & $p$ value & $\mathbf{r}$ & $p$ value \\
\hline \multicolumn{5}{|l|}{ OCT parameters } \\
\hline RNFL average $(\mu \mathrm{m})$ & 0.50 & 0.12 & -0.23 & 0.95 \\
\hline RNFL superior $(\mu \mathrm{m})$ & 0.42 & 0.20 & 0.32 & 0.34 \\
\hline RNFL inferior $(\mu \mathrm{m})$ & 0.50 & 0.12 & -0.42 & 0.90 \\
\hline $\mathrm{CFT}(\mu \mathrm{m})$ & 0.36 & 0.28 & 0.33 & 0.32 \\
\hline Total macular volume $(\mu \mathrm{m})$ & 0.35 & 0.62 & 0.01 & 0.96 \\
\hline \multicolumn{5}{|l|}{ GDx parameters } \\
\hline TSNIT average $(\mu \mathrm{m})$ & -0.53 & 0.09 & -0.60 & 0.05 \\
\hline RNFL superior $(\mu \mathrm{m})$ & -0.40 & 0.22 & -0.43 & 0.06 \\
\hline RNFL inferior $(\mu \mathrm{m})$ & -0.17 & 0.61 & -0.23 & 0.37 \\
\hline NFI & 0.20 & 0.55 & 0.47 & 0.05 \\
\hline \multicolumn{5}{|l|}{ HRT III parameters } \\
\hline Cup area $\left(\mathrm{mm}^{2}\right)$ & -0.30 & 0.38 & -0.38 & 0.22 \\
\hline $\operatorname{Rim}$ area $\left(\mathrm{mm}^{2}\right)$ & 0.09 & 0.77 & 0.20 & 0.51 \\
\hline Rim volume $\left(\mathrm{mm}^{3}\right)$ & 0.19 & 0.57 & 0.14 & 0.63 \\
\hline Cup/Disc area & -0.21 & 0.53 & -0.22 & 0.42 \\
\hline Cup/Disc area (linear) & -0.24 & 0.47 & -0.29 & 0.46 \\
\hline Average cup depth (mm) & -0.23 & 0.50 & -0.35 & 0.51 \\
\hline Maximum cup depth (mm) & -0.11 & 0.75 & -0.49 & 0.13 \\
\hline Cup shape measure & -0.50 & 0.11 & -0.47 & 0.12 \\
\hline RNFL average $(\mu \mathrm{m})$ & -0.34 & 0.30 & -0.22 & 0.51 \\
\hline
\end{tabular}

OCT $=$ Optical coherence tomography, HRT $=$ Heidelberg retina tomograph, GDx $=$ Laser polarimetry, RNFL $=$ Retinal nerve fiber layer, $\mathrm{CFT}=$ Central foveal thickness, TSNIT $=$ Temporal-superior-nasal-inferior-temporal, NFI $=$ Nerve fiber indicator, $\mathrm{MD}=$ median deviation, $\mathrm{PSD}=$ pattern standard deviation. $p<0.05$ indicates statistically significance difference.

ters of the optic disc head and RNFL obtained by using a HRT III, OCT and GDx with global indices of conventional AP to reveal the diagnostic precision of these devices for detecting and following subjects with moderate and severe POAG.

Wollstein et al. [13] suggested that OCT has a closer relationship in glaucomatous progression than VF. In another study Kanamori et al. [14] showed that OCT has the capability to reveal early glaucomatous damage by calculating the RNFL thickness especially in the inferior quadrant. In addition, the RNFL thickness measurements were favorably correlated with the MD. In this study the measurement of inferior RNFL was significantly correlated with MD and PSD in moderate POAG patients. Regarding the severe POAG patients the measurements of superior, inferior, and average RNFL showed statistically significant correlations with MD and PSD. We observed that RNFL thickness obtained by OCT was decreased in the progression of POAG which was well correlated with MD indices. However, there was no significant correlation between OCT measurements and PSD in both moderate and severe POAG patients.

Previous studies demonstrated that macular retinal thickness and volume has been capable of revealing glaucomatous damage, nevertheless peripapillary RNFL thickness measurements showed higher sensitivity and specificity to determine VF abnormalities 
$[15,16]$. In this study macular retinal thickness and volume were significantly different between moderate and severe POAG patients and these measurements were not correlated with MD indices of AP.

In a previous study, Brigatti et al. [17] found a statistically significant correlation between optic disc parameters measured with HRT and functional measurements that were obtained with AP. Wollstein G et al. [18] also compared the confocal laser scanning ophthalmoscope parameters with the MD indices of AP in early glaucomatous patients. They found that MD indices were significantly correlated with the measurements of neuroretinal rim area, optic/disc area, cup/disc area ratio and optic disc area. In our study cup area, cup/disc area, cup/disc area (linear) and maximum cup depth were significantly correlated with MD in moderate POAG. In addition significant correlations were found between cup area, cup/disc area, cup/disc area (linear), average and maximum cup depth and cup shape measure in patients with severe POAG.

Previous studies demonstrated that GDx VCC system can be as sensitive as Stratus-OCT in demonstrating the RNFL thickness decrease in glaucoma. In these studies the NFI was found to be the best discriminating parameter which increases as the glaucoma progresses $[19,20]$. In our study the NFI value was significantly higher in severe POAG than moderate POAG. In addition it was significantly correlated to $\mathrm{MD}$ in severe POAG.

Medeiros FA et al. [3] also evaluated the diagnostic capability of GDx VCC, HRT II, and Stratus OCT and showed similar results for all devices. Zangwill et al. [21] compared the ability of 3 instruments to differentiate healthy eyes from eyes which had early to moderate glaucomatous visual field defects. They found that measurements obtained with OCT and HRT achieved higher sensitivities compared to GDx. In our study OCT achieved the most correlated measurements with AP indices among the three devices in both moderate and severe POAG patients.

DeLeón Ortega et al. [22] found that the measurements of GDx, HRT II and Stratus OCT had high reproducibility in early glaucoma however the repeatability was worse in advanced glaucoma which is probably to conclude the use of NFI by GDx-VCC, rim area, cup area, and cup-to-disc area ratio from both HRT II and Stratus OCT in advanced glaucoma. In our study no significant correlation was found between these imaging devices and AP in patients with MD values less than $-20 \mathrm{~dB}$. This may probably be associated with the loss of glial tissue in the advanced glaucoma which may result the false high measurements.

\section{CONCLUSION}

OCT measurements had the highest correlations with the indices of AP in both moderate and severe POAG patients whereas HRT III had the lowest values. The correlations of all devices decreased in patients with more advanced glaucomatous damage hence AP still seems to be useful in the follow up disease progression in these glaucoma patients.

\section{Authors' Contribution}

Study Conception: MEA, MB, EG, ÖBT, SY; Study Design: MEA, MB, EG, ÖBT, SY; Supervision: MEA, MB, EG, ÖBT, SY; Funding: MEA, MB, EG, ÖBT, SY; Materials: MEA, MB, EG; Data Collection and/or Processing: MEA, MB, EG; Statistical Analysis and/or Data Interpretation: MEA, MB, EG; Literature Review: MEA, MB, EG; Manuscript Preparation: MEA, MB, EG and Critical Review MEA, MB, EG.

\section{Conflict of interest}

The authors disclosed no conflict of interest during the preparation or publication of this manuscript.

\section{Financing}

The authors disclosed that they did not receive any grant during the conduction or writing of this study.

\section{REFERENCES}

1. Boden C, Hoffmann EM, Medeiros FA, Zangwill LM, Weinreb $\mathrm{RN}$, Sample PA. Intereye concordance in locations of visual field defects in primary open-angle glaucoma: diagnostic innovations in glaucoma study. Ophthalmology 2006;113:918-23.

2. Garway-Heath DF. Early diagnosis in glaucoma. Prog Brain Res 2008;173:47-57.

3. Medeiros FA, Zangwill LM, Bowd C, Weinreb RN. Comparison of the GDx VCC scanning laser polarimeter, HRT II confocal scanning laser ophthalmoscope, and stratus OCT optical coherence tomograph for the detection of glaucoma. Arch Ophthalmol 2004;122:827-37.

4. Hoh ST, Greenfield DS, Mistlberger A, Liebmann JM, 
Ishikawa H, Ritch R. Optical coherence tomography and scanning laser polarimetry in normal, ocular hypertensive, and glaucomatous eyes. Am J Ophthalmol 2000;129:129-35.

5. Kerrigan-Baumrind LA, Quigley HA, Pease ME, Kerrigan DF, Mitchell RS. Number of ganglion cells in glaucoma eyes compared with threshold visual field tests in the same persons. Invest Ophthalmol Vis Sci 2000;41:741-8.

6. Nomoto H, Matsumoto C, Takada S, Hashimoto S, Arimura E, Okuyama S, et al. Detectability of glaucomatous changes using SAP, FDT, flicker perimetry, and OCT. J Glaucoma 2009; 18:16571.

7. Greaney MJ, Hoffman DC, Garway-Heath DF, Nakla M, Coleman AL, Caprioli J. Comparison of optic nerve imaging methods to distinguish normal eyes from those with glaucoma. Invest Ophthalmol Vis Sci 2002;43:140-5.

8. Anderson DR, Patella VM, eds. Automated Static Perimetry. 2nd ed. St. Louis: Mosby. 1998.

9. Bengtsson B, Heijl A. Evaluation of a new perimetric threshold strategy, SITA, in patients with manifest and suspect glaucoma. Acta Ophthalmol Scand 1998;76:268-72.

10. Leung CK, Ye C, Weinreb RN, Cheung CY, Qiu Q, Liu S, et al. Retinal nerve fiber layer imaging with spectral-domain optical coherence tomography a study on diagnostic agreement with Heidelberg Retinal Tomograph. Ophthalmology 2010;117:267-74.

11. Jaffe GJ, Caprioli J. Optical coherence tomography to detect and manage retinal disease and glaucoma. Am J Ophthalmol 2004; 137:156-69.

12. Weinreb RN, Bowd C, Zangwill LM. Scanning laser polarimetry in monkey eyes using variable corneal polarization compensation. J Glaucoma 2002;11:378-84.

13. Wollstein G, Schuman JS, Price LL, Aydin A, Stark PC, Hertzmark E, et al. Optical coherence tomography longitudinal evaluation of retinal nerve fiber layer thickness in glaucoma. Arch Ophthalmol 2005;123:464-70.

14. Kanamori A, Nakamura M, Escano MF, Seya R, Maeda H,
Negi A. Evaluation of the glaucomatous damage on retinal nerve fiber layer thickness measured by optical coherence tomography. Am J Ophthalmol 2003;135:513-20.

15. Ojima T, Tanabe T, Hangai M, Yu S, Morishita S, Yoshimura $\mathrm{N}$. Measurement of retinal nerve fiber layer thickness and macular volume for glaucoma detection using optical coherence tomography. Jpn J Ophthalmol 2007;51:197-203.

16. Wollstein G, Schuman JS, Price LL, Aydin A, Beaton SA, Stark PC, et al. Optical coherence tomography (OCT) macular and peripapillary retinal nerve fiber layer measurements and automated visual fields. Am J Ophthalmol 2004;138:218-25.

17. Brigatti L, Caprioli J. Correlation of visual field with scanning confocal laser optic disc measurements in glaucoma. Arch Ophthalmol 1995;113:1191-4.

18. Wollstein G, Garway-Heath DF, Hitchings RA. Identification of early glaucoma cases with the scanning laser ophthalmoscope. Ophthalmology 1998;105:1557-63.

19. Reus NJ, Lemij HG. Diagnostic accuracy of the GDx VCC for glaucoma. Ophthalmology 2004;111:1860-5.

20. Leung CK, Chong KK, Chan WM, Yiu CK, Tso MY, Woo J, et al. Comparative study of retinal nerve fiber layer measurement by StratusOCT and GDx VCC, II: structure/function regression analysis in glaucoma. Invest Ophthalmol Vis Sci 2005;46:370211.

21. Zangwill LM, Bowd C, Berry CC, Williams J, Blumenthal EZ, Sanchez-Galeana CA, et al. Discriminating between normal and glaucomatous eyes using the Heidelberg Retina Tomograph, GDx Nerve Fiber Analyzer, and Optical Coherence Tomograph. Arch Ophthalmol 2001;119:985-93.

22. DeLeon Ortega JE, Sakata LM, Kakati B, McGwin G Jr, Monheit BE, Arthur SN, et al. Effect of glaucomatous damage on repeatability of confocal scanning laser ophthalmoscope, scanning laser polarimetry, and optical coherence tomography. Invest Ophthalmol Vis Sci 2007;48:1156-63. 